\title{
Role of Magnetic Resonance Imaging in the Evaluation of Basal Ganglia Lesions
}

\author{
Diddy Vamshi Kiran ${ }^{1}$, Rama Krishna Rao Baru \\ ${ }^{1}$ Post Graduate, ${ }^{2}$ Professor, Department of Radio-Diagnosis, Narayana Medical College and Hospital, Nellore - 524002 , \\ Andhra Pradesh, India
}

Corresponding author: Dr Ramakrishna B, Department of Radio-Diagnosis, Narayana Medical College and Hospital, Nellore - 524 002, Andhra Pradesh, India

DOI: $10.21276 / \mathrm{ijcmsr} .2018 .3 .3 .13$

How to cite this article: Diddy Vamshi Kiran, Rama Krishna Rao Baru. Role of magnetic resonance imaging in the evaluation of basal ganglia lesions. International Journal of Contemporary Medicine Surgery and Radiology. 2018;3 (3):C59-C65.

\section{A B S T R A C T}

Introduction: Radiologists will encounter bilaterally symmetrical abnormalities of the basal ganglia, usually in diffuse systemic and metabolic diseases. Study aimed to identify the spectrum of diseases involving basal ganglia disease and also to evaluate other sites of involvement in these patients.

Material and methods: 30 patients referred to our hospitalwith suspicion of basal ganglia lesions subjected to MR examinations by 3TMR system using a 32-channel phased array head coil.

Results: Most common etio-pathologies affecting basal ganglia are metabolic diseases (67\%), Japanese encephalitis and dengue encephalitis (17\%), Neoplasm's Glioma and DNET (10\%) and degenerative NBIA and Huntington's disease (7\%) respectively. Among metabolic diseases Hypoxic ischemic encephalopathy (30\%) and Leigh's syndrome (30\%) are the most common entities followed by hypoglycemic encephalopathy (10\%) and posterior reversible encephalopathy syndrome (10\%) diagnosed respectively. Among the Thirty patients with basal ganglia lesions, bilateral symmetrical involvement in 22 cases (73\%), unilateral involvement in 5 cases (17\%) and bilateral asymmetrical involvement in 3 cases (10\%). There are 5 cases with Caudate +Putamen (Dorsal striatum), 4 cases with Putamen+globus pallidus (lentiform nucleus) and 7 cases withentire basal ganglia (Corpus striatum) involvement in metabolic disease patients.

Conclusion: MR imaging has dramatically improved the ability to visualise deep grey structures of basal ganglia and spectrum.

Key words: Basal Ganglia Lesions, Magnetic Resonanceimaging, Huntington's Disease.

\section{INTRODUCTION}

The deep grey matter structures of the basal ganglia comprise the caudate nucleus, putamen and globus pallidus. Computed tomography (CT) and magnetic resonance (MR) imaging have dramatically improved the ability to visualize the deep gray structures of the basal ganglia ${ }^{1}$. They form the key components of the extrapyramidal motor system, and receive projections from almost every region of the cerebral cortex, playing a vital role in integrating movement ${ }^{2}$.

MRI allows detailed visualization of the morphology, signal intensity and metabolic content of the DGM nuclei, together with visualization of normal cortical development and normal myelination of the white matter.

The basal ganglia have high energy adenosine triphosphate (ATP) produced by oxidative phosphorylation within the mitochondria] requirements, increased blood flow and are rich in neurotransmitters and trace metals such as iron, copper and manganese. Hence, they are vulnerable to any systemic disease or generalised process that alters cerebral metabolism, which can lead to selective damage to the basal ganglia. However, damage to the deep grey matter nuclei may be visualised either as basal ganglia lesions in isolation or as part of more generalised brain damage also involving other grey or white matter structures ${ }^{2}$.

Among the many causes of basal ganglia damage, bilateral symmetrical lesions typically are caused by diffuse systemic or metabolic conditions. Often, the cause (for example hypoxic ischemic encephalopathy in the context of cardiac arrest) is readily evident, but sometimes, the radiologist is faced with situations of bilateral symmetrical lesions in the basal ganglia for initial diagnosis (for example, coma of unknown cause) before the results of relevant investigations are known.

There is considerable variation and overlap in both the clinical and radiologic features of abnormalities affecting the deep gray matter nuclei. Hence, no classification scheme is fool proof ${ }^{2}$.

The systematic approach to this uncommon group of systemic and metabolic conditions is often challenging, but assessment of all the neuroimaging findings (not merely of the basal ganglia but other features) is an essential component for final diagnosis and sometimes prognosis ${ }^{2}$.

The neuroimaging diagnosis is influenced not only by detection of specific MR imaging features such as restricted diffusion and the presence of haemorrhage, but also by detection of abnormalities involving other parts of the brain, 
especially the cerebral cortex, brainstem, and white matter. Judicious use of confirmatory neuroimaging investigations, especially diffusion-weighted imaging, MR angiography, MR venography, and MR spectroscopy during the same examination, may help improve characterization of these abnormalities and help narrow the differential diagnosis ${ }^{3}$.

Recognition and correct evaluation of basal ganglia abnormalities, together with a proper clinical history and laboratory findings, may enable the identification of spectrum of disease entities and lead to earlier diagnosis ${ }^{4}$.

Reaching a diagnosis in the early stages of acute diseases in many patients is crucial for instigating prompt specific therapy leading to a favourable outcome ${ }^{5}$.

The neuroradiologist can thus play an important role in contributing imaging features to the overall clinical, biochemical and genetic picture that makes up an accurate picture of patients with systemic and metabolic disease ${ }^{2}$.

The aim of the study was to evaluate the spectrum of pathologic conditions of basal ganglia involvement (Excluding vascular pathologies), and the radiological assessment of these conditions with clinical correlation.

\section{MATERIAL AND METHODS}

Patients attending the department of Radio-diagnosis, Narayana Medical College, Nellore were the main source of data for this study. The study was approved by the institutional ethical committee and study details were explained to all the cases or relatives and consent was obtained. All patients referred to the department of Radiology with clinical suspicion of basalganglia (deep grey matter) lesions in a period of 2yrs from SEP 2015 to SEP 2017 will be subjected for study. Total 30 patients with basal ganglia involvement were included based on inclusion creteria.

Inclusion Criteria: Patients with extrapyramidal symptoms, patients with suspected metabolic encephalopathy, all patients with incidentally diagnosed basal ganglia lesions by CT, follow up patients of basal ganglia lesions, cases of all age groups irrespective of sex.

Exclusion Criteria: Vascular pathologies of basal ganglia (Infarcts and haemorrhages), Patient having history of claustrophobia, Patient having history of metallic implants insertion, cardiac pacemakers and metallic foreign body in situ.

Equipment: The MR examinations were performed on all patients who met inclusion criteria at a $3 \mathrm{~T}$ whole-body MR system (DISCOVERY 750W 3T MRI GE Healthcare, Chicago, Illinois) using a 32-channel phased array head coil.

Sequences: Conventional spin echo sequences, axial T1, T2 and FLAIR: Coronal FLAIR, DWI; SWAN and Postcontrast $\mathrm{T} 1+\mathrm{C}$ if required.

Special sequences such as Single voxel spectroscopy was performed at TE of 33 and $144 \mathrm{msec}$ were used as and when required.

\section{STATISTICAL ANALYSIS}

Statistical analysis was performed using percentages and proportionswith the help of microsoft office 2015.

\section{RESULTS}

Total 30 patients were evaluated with basal ganglia

\begin{tabular}{|c|c|c|c|c|c|c|}
\hline Pathology & Caudate only & $\begin{array}{c}\text { Caudate + } \\
\text { Putamen }\end{array}$ & $\begin{array}{c}\text { Putamen } \\
\text { only }\end{array}$ & $\begin{array}{c}\text { Putamen + } \\
\text { GP }\end{array}$ & GP only & $\begin{array}{c}\text { Corpus } \\
\text { striatum }\end{array}$ \\
\hline HIE & 1 & 1 & 1 & 1 & - & 2 \\
\hline Leigh's syndrome & - & 1 & - & 1 & - & 4 \\
\hline Hypoglycemic encephalopathy & - & - & - & 1 & - & 1 \\
\hline Hepatic encephalopathy & - & - & - & - & 1 & - \\
\hline PRES & - & 1 & - & 1 & - & - \\
\hline MSUD & - & - & - & - & 1 & - \\
\hline Osmotic myelinolysis & - & 1 & - & - & - & - \\
\hline Wilson's disease & - & 1 & - & - & - & - \\
\hline
\end{tabular}

\begin{tabular}{|c|c|c|c|c|c|}
\hline Pathology & $\begin{array}{l}\text { Basal ganglia + } \\
\text { Thalamus }\end{array}$ & $\begin{array}{l}\text { Basal ganglia + } \\
\text { Cortex }\end{array}$ & $\begin{array}{l}\text { Basal ganglia + } \\
\text { White matter }\end{array}$ & $\begin{array}{c}\text { Basal ganglia + } \\
\text { Brainstem }\end{array}$ & $\begin{array}{c}\text { Basal ganglia + } \\
\text { Cortex + White } \\
\text { matter }\end{array}$ \\
\hline HIE & 5 & 6 & 2 & - & 2 \\
\hline Leigh's syndrome & 2 & - & - & 5 & - \\
\hline $\begin{array}{l}\text { Hypoglycemic encephalop- } \\
\text { athy }\end{array}$ & - & 2 & 2 & - & 2 \\
\hline Hepatic encephalopathy & - & - & - & - & - \\
\hline PRES & - & 1 & 2 & - & 1 \\
\hline MSUD & 1 & 1 & & 1 & - \\
\hline Osmotic myelinolysis & - & - & - & 1 & - \\
\hline Wilson's disease & 1 & - & - & 1 & - \\
\hline
\end{tabular}



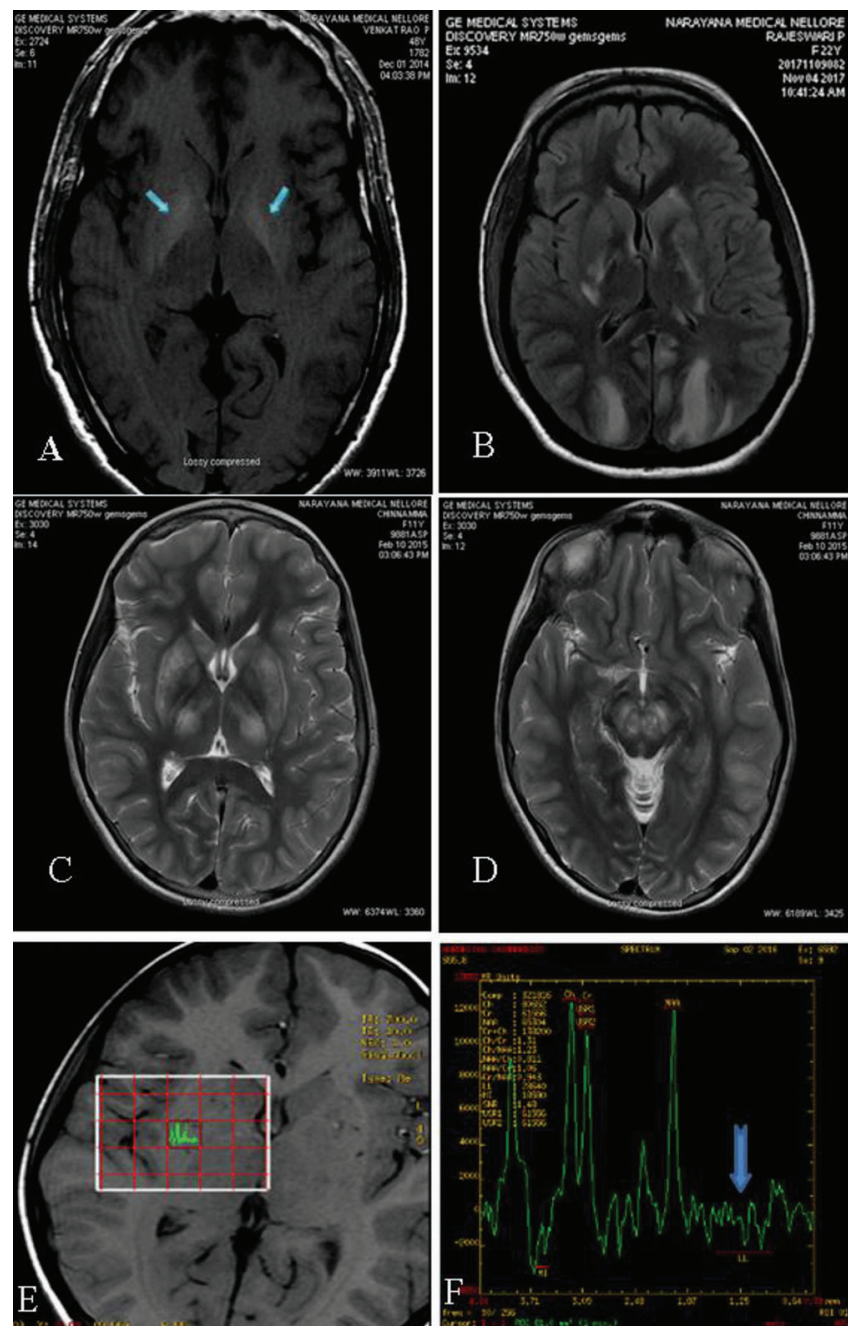

Figure-1: A. Axial T1WI of brain show symmetrical hyper intensities in the bilateral Globi Pallidi and substania nigra in a decompensated hepatic failure patient -1 (HE), B. Axial FLAIR reveals symmetrical areas of FLAIR hyper intensity with diffusion restriction involving cortical and sub cortical white matter of bilateral occipital lobe and right caudate and putamen in hypertensive patient-2 (PRES). C and D. Axial T2 and FLAIR images of brain show bilaterally symmetric $\mathrm{T} 2$ hyper intensities in the ventrolateral thalamus,caudate and putamina (Wilson's). E and F. MRS shows inverted lipid-lactate peak at1.1PPM (Leigh's).

involvement. Thirty patients were evaluated, whose age group ranged from 1 to $>60$ years. The highest incidence of basal ganglia lesions were found in 21-30 years age group accounting for $23 \%$ of cases and least was seen in age group of $41-50$ years constituting $3 \%$. Thirty patients were evaluated of which $18(60 \%)$ were males and $12(40 \%)$ were females.

Out of the 30 patients who were evaluated, Metabolic diseases accounts for 20 cases $(67 \%)$ and is the most common pathology followed by infectious diseases representing 5 cases (17\%), Neoplasm's accounting for 3 cases (10\%) and degenerative diseases involving 2 cases (7\%).

Among metabolic diseases, there are 6 cases each with Hypoxic ischemic encephalopathy and Leigh's syndrome, 2 cases each with hypoglycemic encephalopathy, posterior reversible encephalopathy syndrome and 1 case each with Maple syrup urine disease, Hepatic encephalopathy, Wilson's disease, Extrapontine myelinolysis.
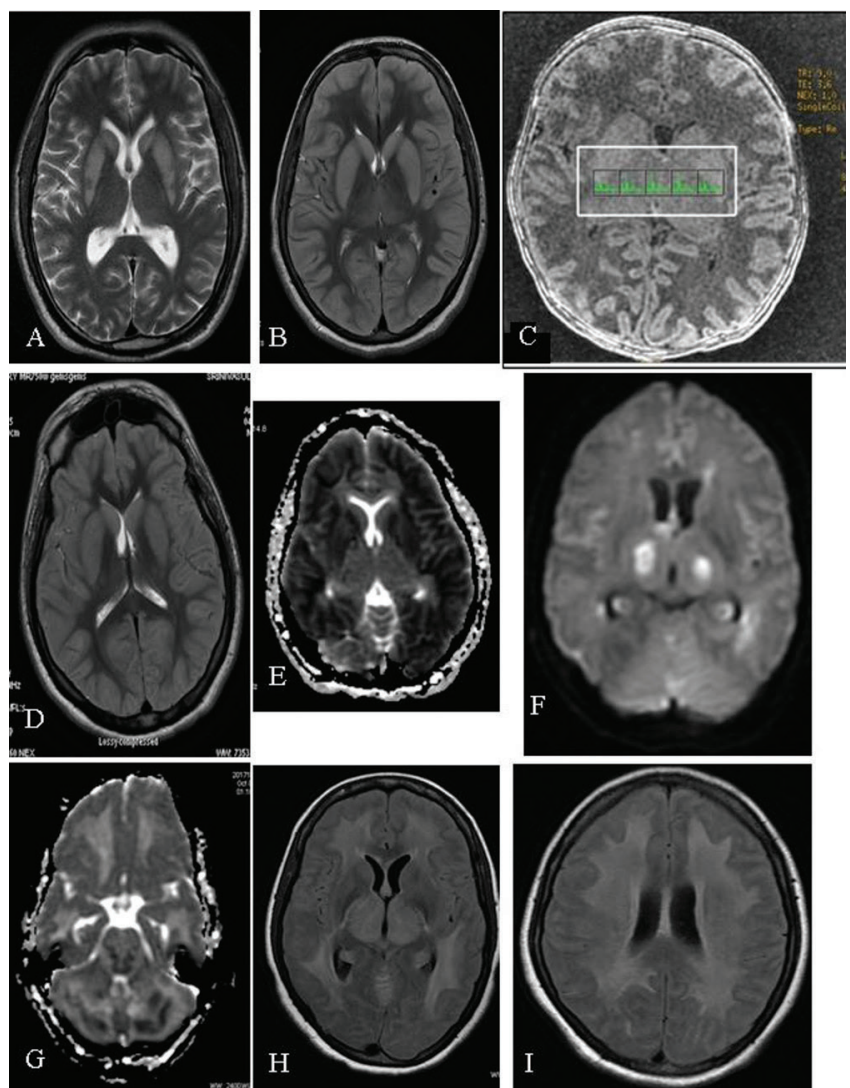

Figure-2: A. Axial T2W MR show bilaterally symmetric hyper intense areas in caudate nucleus, putamen and in central pons with peripheral tegmental sparing termed as "Bat wing appearance"/"Trident sign" in a elderly female following rapid electrolyte correction (Extrapontine myelinolysis). B.Axial T2 shows bilateral symmetrical involvement of caudate nuclei, putamen, cortical and sub cortical white matter of cerebral hemisphere with typical sparing of thalamus in a diabetic patient (Neuroglycopenia). C. Axial T1w shows bilateral symmetrical T1 Iso to hypo intensity showing diffusion restriction with relatively crisp margins in globus pallidi, posterior limb of internal capsule, dorsal brainstem, cerebral peduncles and cerebellum. MRS shows a peak at $0.9 \mathrm{ppm}$ corresponding to branched chain amino acid (Valine) appreciable in SV MRS done at TE $35 \mathrm{~ms}$ (Maple syrup urine). D and E. Axial T2W, Axial ADC sequence images shows bilateral symmetrical T2hyperintensities involving caudate nuclei, putamen, thalami and swollen cortical gyri of bilateral cerebral hemisphere in post cardiac arrest patient (Hypoxic-Ischemic Encephalopathy). F. Axial DWI images with corresponding ADC map shows diffusion restriction in bilateral globus pallidi,thalami,brainstem and cerebellar hemisphere. G, H and I. Axial SWI image shows haemorrhage in bilateral thalami. Axial FLAIR sequence shows diffuse deep white matter edema involving bilateral fronto-parietal lobes and corona radiate (Viral Encephalitis).

Among thirty patients with basal ganglia lesions, seizures are the most common presentation followed by altered mental state especially in acute cases like metabolic and infectious diseases. Extrapyramidal symptoms (Gait disturbances, abnormal dancing movement, hypertonia, dystonia, rigidity etc.) are the common presentation in chronic cases like degenerative and neoplasm's.

Incidence of 20 cases diagnosed with metabolic, 5 cases of 
infectious, 3 cases of neoplasms, and cases of degenerative diseases respectively. 22 cases identified to be bilateral symmetrical side of pathology in basal ganglia lesions.

Among Thirty patients, 24 (80\%) cases showed diffusion restriction (partial/complete) and 6 (20\%) cases showed no restriction.

Out of Thirty patients evaluated, metabolic diseases are seen in 20 cases (67\%). Among the 20 cases Males were 11 and Females were 9.Bilateral symmetrical involvement of basal ganglia is seen in 19 cases (95\%) and unilateral involvement is noted in 1 case (5\%).

Most common primary basal ganglia nuclei involved was caudate + lentiform nucleus (Corpus striatum) in 7 cases (35\%), followed by Caudate + Putamen in 5 cases $(25 \%)$, Putamen+GP (Lentiform nucleus) in 4 cases (20\%), only GP in 2 cases (10\%), only putamen in 1 case (5\%) and lastly only caudate in 1 case (5\%) (Table 1) (Figure 1). Involvement of most common site of neuroparenchyma in addition to basal ganglia includes BG + Thalamus in 9 cases (45\%), followed by $\mathrm{BG}+$ Cortex in 10 cases $(50 \%), \mathrm{BG}+$ Cortex + White matter in 5 cases $(25 \%), \mathrm{BG}+$ White matter in 6 cases $(30 \%)$ and finally BG + Brainstem in 8 cases (40\%) (Table 2) (Figure 2).

\section{DISCUSSION}

Magnetic resonance imaging is a non-invasive, multiplanar and highly accurate method with better inherent contrast that demonstrates the lesion accurately. MRI provides an accurate assessment of the brain changes in various spectrum of basal ganglia lesion. This was a Hospital based prospective study done in the Department of Radio diagnosis and Imaging, Narayana medical college and hospital, aimed at studying the MR appearances/patterns in various spectrums of Basal ganglia lesions. In our study of MR imaging of basal ganglia lesions, we evaluated 30 patients. Out of the 30 patients who were evaluated, Metabolic diseases accounts for 20 cases $(67 \%)$ and is the most common pathology followed by infectious diseases representing 5 cases (17\%), Neoplasm's accounting for 3 cases (10\%) and Degenerative diseases involving 2 cases (7\%). Finelli et al, ${ }^{6}$ in his case series study stated, metabolic conditions were the most common cause of acute bilateral symmetric DGMN lesions.

Among metabolic diseases HIE (30\%) and Leigh's syndrome $(30 \%)$ are the most common entities followed by hypoglycemic encephalopathy (10\%) and PRES (10\%). Hepatic encephalopathy, Wilson disease, Maple syrup urine disease and extrapontine myelinolysis are other fewer pathologies accounting for rest of the metabolic diseases (20\%). Viral encephalitis like Japanese encephalitis and dengue encephalitis are the most common pathologies under infectious diseases. Glioma and DNET are the pathologies included under Neoplasm's. NBIA and Huntington's disease are the disease entities which represents the degenerative diseases. Among thirty patients with basal ganglia lesions, seizures are the most common presentation followed by altered mental state especially in acute cases like metabolic and infectious diseases Extrapyramidal symptoms (Gait disturbances, abnormal dancing movement, hypertonia, dystonia, rigidity etc,) are the common presentation in chronic cases like degenerative and neoplasm's. Among the Thirty patients with basal ganglia lesions, bilateral symmetrical involvement noted in 22 cases (73\%), unilateral involvement noted in 5 cases $(17 \%)$ and bilateral asymmetrical involvement noted in 3 cases (10\%).

Among Thirty patients who were evaluated; 24 (80\%) cases showed diffusion restriction (partial/complete) and 6 (20\%) cases showed no restriction. Out of thirty patients, metabolic diseases are seen in 20cases (67\%). Among the 20 cases Males were11 and Females were 9.

Bilateral symmetrical involvement of basal ganglia is seen in 19 cases (95\%) and unilateral involvement is noted in 1 case (5\%).

Most common primary basal ganglia nuclei involved was caudate + lentiform nucleus (Corpus striatum) in 7 cases (35\%), followed by Caudate +Putamen in 5 cases $(25 \%)$, Putamen+GP (Lentiform nucleus) in 4 cases (20\%), only GP in 2 cases (10\%), only putamen in 1case (5\%) and lastly only caudate in 1 case $(5 \%)$.

Involvement of most common site of neuroparenchyma in addition to basal ganglia includes BG + Thalamus in 9 cases (45\%), followed by BG + Cortex in10 cases (50\%), BG + Cortex + White matter in 5 cases $(25 \%), \mathrm{BG}+$ White matter in 6 cases (30\%) and finally BG + Brainstem in 8 cases (40\%). Diffusion restriction was noted in 17 cases (85\%) and in 3 cases (15\%) no diffusion restriction is seen.Among 20 cases,various pathologies constitute the metabolic diseases. The most common entity among the metabolic diseases are HIE (6 cases, 30\%) and Leigh's syndrome (6 cases, 30\%). $\mathrm{HIE}$ is the most common metabolic disease seen especially in adults.

Cardiac arrest was the mechanism of injury in the majority of the patients of our study population (5 cases). Cardiac causes accounted for 16 out of 39 cases of hypoxic-ischemic insult in the study by Choi et al, ${ }^{7}$ and 14 out of 22 cases in the study by Topcuoglu et $\mathrm{al}^{8}$.

Studies by Wu et al, ${ }^{9}$ Arbelaez et al, ${ }^{10}$ were focused only on patients with cardiac arrest as the cause of hypoxic-ischemic insult.

Involvement of cerebral cortex (6 cases, 100\%) was noted in association with basal ganglia noted in all cases. Thalamus involvement in addition to basal ganglia involvement is seen in 4 cases (67\%).Hippocampal involvement in association with basal ganglia involvement noted in 3 cases (50\%).

Diffuse cortical and deep grey matter pattern with perirolandic involvement was the most frequent pattern across the major mechanism in a study by Eluvathingal Muttikkalet al. ${ }^{8}$

Choi ${ }^{7}$ et al, in their study of 39 patients categorized the brain injuries into four patterns: normal, isolated cortical injury, isolated deep grey nuclei injury, and mixed injuries (cortex and deep grey).

Wu et al, ${ }^{9}$ concluded that whole-brain median ADC was a predictor of poor outcome.

Leigh's syndrome is next common entity among metabolic diseases which is exclusively seen in pediatric population especially neonates presenting with complaints of not accepting feeds, decreased activity, neuronal regression and seizures.

Medulla was normal without any hypertrophic olivary degeneration in our study.Brainstem and basal ganglia 
(Putamen) involvement was seen in 4 Leigh syndrome children with novel SURF1 mutation in a study by Kothari Sonam, Nahid Akthar Khan et al, ${ }^{11}$.

Eliana Bonfante and Mary Kay Koenig et al,12 described the neuroimaging findings in a cohort of 17 children with genetically confirmed Leigh syndrome.

MRS was done in 2 cases with spectral mapping over putamen at $144 \mathrm{~ms}$ which revealed Lipid lactate peak at 1.1 PPM. Of 6 cases, Lab investigation reveals metabolic acidosis in 4 cases with increased Serum lactate levels.

Diffusion restriction was noted in both cases. Follow up study of one patient after 1 month revealed normal neuroparenchyma suggesting reversible insult.Serial MRI evaluation of 4 vegetative patients with profound hypoglycemia revealed signal intensity changes in caudate and lenticular nuclei, cerebral cortex, substantia nigra, and/or hippocampus from 8 days to 12 months after onset in a study by Masayuki Fujioka, et $\mathrm{al}^{13}$.

Tchoyoson Lim et al, ${ }^{14}$ evaluated 7 patients with profound hypoglycemia, cerebral cortex involvement was seen in all cases and basal ganglia involvement was seen in 2 cases and are had poor outcome.

PRES (Posterior reversible encephalopathy syndrome) is the other entity accounting for $11 \%$ (2 cases).

Bartynski et al, ${ }^{15}$ evaluated large cohort of 136 patients who experienced PRES to comprehensively assess the imaging patterns: Vasogenic edema was consistently present in the parietal or occipital regions (98\%), but other locations were common including the frontal lobes $(68 \%)$, inferior temporal lobes (40\%), and cerebellar hemispheres (30\%).

Extrapontine myelinolysis accounts for 1 case (5\%). Elderly female presented to casualty with gastroenteritis, considering the clinical condition patient has been vigorously treated with IV fluids following which patient had altered sensorium.

Gary M. Miller et al, ${ }^{16}$ reviewed 9 patients with CPM, all patients had basipontis involvement and extrapontine myelinolysis, were identified in three of the patients in the peniventricular white matter, basal ganglia, and corticomedullary junction bilaterally.

Sabale Avinash et al, ${ }^{17}$ reported two cases of patients with proven malignancy who developed extrapontine myelinolysis after treatment for hyponatremia.An early MRI of the brain in suspected/high-risk cases of osmotic myelinolysis may show features of extrapontine myelinolysis in the form of bilateral basal ganglia hyper intensity on T2W and FLAIR images and diffusion restriction with typical pallidal sparing. Hepatic encephalopathy accounts for 5\% (1-case).

Etsuo Inoue et al, ${ }^{18}$ evaluated Sixteen patients with cirrhosis of the liver underwent cranial magnetic resonance (MR) imaging and transarterial portography to evaluate the relationship between basal ganglia lesions and portalsystemic collateral vessels.

A Pujol et $\mathrm{al}^{19}$ evaluated Seventy-seven patients with chronic liver disease, candidates for orthotopic liver transplantation with MRI The high-intensity signal in the globus pallidus on T1w MRI was observed in 58 (75\%) patients.

Ailan Cheng et al ${ }^{20}$ retrospectively studied 10 MSUD patients confirmed by genetic testing. 6 cases are of classic form the classic form of MSUD involved the basal ganglia in six cases; the cerebellum, mesencephalon, pons, and supratentorial area in five cases; and the thalamus in four cases, respectively.

Felber et al, ${ }^{21}$ demonstrated elevation of lactic acid, and presence of a peak at $0.9-1.0 \mathrm{ppm}$, belonging to methyl resonance of branched chain amino acids.

Wilson's disease is another rare entity accounting for 1 case (5\%). Paediatric female child who has hepatic failure presented with psychomotor illness and movement disorder. Laboratory investigation revealed copper traces in urine. On MRI evaluation, there is bilateral symmetrical T2 and FLAIR hyper intensity involving lentiform nucleus, thalamus, midbrain and pontine tegmentum without diffusion restriction.

T.J. Kim,I.O. Kimet al, ${ }^{22}$ evaluated a cohort of pediatric Wilson disease patients in whom, the lentiform nuclei are involved most often, followed by the thalami, pons, midbrain, superior and middle cerebellar peduncles, and cerebellar nuclei.

Bilateral asymmetrical involvement was seen in 3 cases (60\%), unilateral involvement and bilateral symmetrical involvement noted each in 1 case20\%).Diffusion restriction is seen in all cases100\%). Thalamus involvement is seen in all cases (100\%) with associated haemorrhage was seen in 2 cases (40\%). Brainstem, cerebellum and deep white matter involvement was seen in 2 cases (40\%). Basal cisterns, ventricles and meningeal enhancement are normal in all cases.Serology of 2 patients among encephalitis picture is positive for Dengue virus. The findings in rest of the 4 patients of our study reveals clinical features of encephalitis with negative dengue serology test and MRI imaging pattern shows deep grey matter and thalamic involvement which suggest the probability of $\mathrm{JE}$ and is considered in these 4 patients.J. Kalita et. al, ${ }^{23}$ evaluated forty two patients with JE. Thalamic lesions on CT and/or MRI combined had sensitivity 23\% (95\% confidence interval 12.9-33.1\%), specificity 100\%, positive predictive value $100 \%$ and negative predictive value $42.1 \%$ (95\% confidence interval 30.2-53.8\%) for a diagnosis of JE in a cohort of 75 patients with suspected encephalitis in a study by N. M. Dung and Lance Turtle etal, (2009) ${ }^{24}$.

U.K. Misraa, J. Kalitaa, ${ }^{25}$ et.al, evaluated 88 consecutive viral encephalitis patients, of them 22 patients had JE, 9 had dengue, 8 had herpes simplex encephalitis (HSE), 2 had Epstein-Barr virus encephalitis (EBVE) and 47 had nonspecific encephalitis.

Sanjeev Kumar etal, ${ }^{26}$ evaluated twenty-one serologically confirmed patients of dengue with altered sensorium who underwent MRI in them was 20. MRI was abnormal in 9 (45\%).

SoniSR Das et al, ${ }^{27}$ evaluated magnetic resonance imaging (MRI) findings of 3 patients with dengue fever diagnosed by positive dengue NS1 antigen revealed nonspecific imaging features of dengue encephalitis in two cases and dengue meningoencephalitis in one case in the form of diffuse cerebral oedema, bilateral symmetrical FLAIR and T2 hyper intensities in thalami, pons, and medulla with heterogenous or peripheral enhancement

José M. García-Santos ${ }^{28}$ etal, described among brain tumours, those arising from the deep brain are rare. In many cases they 
are low-grade astrocytomas followed by germ cell tumours. Wei Fu et $\mathrm{al}^{29}$ retrospectively analyzed 35 children with PBGRT fromDecember 2011to December 2015. Most common were 15 astrocytomas and 11 germ cell tumours (GCT).

DNET (Dysembryoplastic neuroepithelial tumour) is the other neoplasm seen in our study. Young adult patient presented with recurrent history of partialseizures.

No obvious perilesional edema noted. Associated cortical dysplasia or cortical tubers are not seen in our study.On T1+ $\mathrm{C}$ contrast study, no obvious enhancement of lesion seen. Clinico-radiologic criteria for the diagnosis of DNET ${ }^{30}$ are seen in our case.

These findings were very much similar to our study result of DNET.Degenerative diseases are rare ones involving basal ganglia accounting for 2 cases (7\%)in my study. These diseases are confined only to basal ganglia nuclei without involvement of rest of the neuroparenchyma.Bilateral symmetrical involvement without obvious diffusion restriction is seen. Among the two cases, first one was NBIA (Previously known as Hallevordan Spatz disease) was seen in a $25 \mathrm{yrs}$ old male presenting with movement disorder.

On imaging, there was bilateral symmetrical globus pallidus involvement showing T1, T2 and SWAN hyper intensity with surrounding hypo intensity appearing as "Eye of Tiger" sign without diffusion restriction.

These findings were very much similar to our study result in NBIA. Second degenerative disease included in my study was Huntington's disease, seen in a 40 yrs old male presenting with involuntary dancing movements (chorea).

On evaluation, exclusive bilateral caudate and putamen (Caudate >Putamen) nucleus involvement noted in the form of atrophy. On axial images frontal horn of bilateral lateral ventricles appears as "Box Car" configuration.

\section{CONCLUSION}

Magnetic Resonance Imaging has dramatically improved the ability to visualise deep grey structures of basal ganglia and spectrum of diseases involving it. Basal ganglia has a characteristic appearance on MR sequences and abnormalities in their appearance have a characteristic pattern which helps in narrowing the differential diagnosis. Magnetic resonance imaging can contribute greatly to arriving at correct diagnosis with available clinical and laboratory data. Magnetic resonance imaging is useful in monitoring disease progression. It also has a prognostic implication.

\section{REFERENCES}

1. Ho VB, Fitz CR, Chuang SH, Geyer CA. Bilateral basal ganglia lesions: pediatric differential considerations. Radiographics 1993;13 (1):26992.

2. Lim CC. Magnetic resonance imaging findings in bilateral basal ganglia lesions. Annals of the Academy of Medicine, Singapore 2009;38 (2):795-8.

3. Hegde AN, Mohan S, Lath N, et al. Differential diagnosis for bilateral abnormalities of the Basal Ganglia and thalamus. RadioGraphics 2011;31:5e30.

4. Zuccoli G, Yannes MP, Nardone R, Bailey A, Goldstein A. Bilateral symmetrical basal ganglia and thalamic lesions in children: an update. Neuroradiology. 2015;57 (10):973-89.

5. Quattrocchi CC,Longo D, Delfino LN, ErranteY,Aiello C, Fariello G, Bernardi B. MR differential diagnosis of acute deep grey matter pathology in paediatric patients. Pediatric radiology. 2013;43 (6):743-61.

6. Finelli PF, DiMario Jr FJ. Diagnostic approach in patients with symmetric imaging lesions of the deep gray nuclei. The neurologist. 2003;9 (5):250-61.

7. Choi SP, Park KN, Park HK, Kim JY, Youn CS, Ahn KJ, Yim HW. Diffusion-weighted magnetic resonance imaging for predicting the clinical outcome of comatose survivors after cardiac arrest: a cohort study. Critical care. 2010;14 (1):R17.

8. Muttikkal TJ, Wintermark M. MRI patterns of global hypoxic-ischemic injury in adults. Journal of Neuroradiology. 2013;40 (3):164-71.

9. Wu O, Sorensen AG, Benner T, et al. Comatose patients with cardiac arrest: Predicting clinical outcome with diffusionweighted MR imaging. Radiology 2009;252:173-81.

10. Arbelaez A, Castillo M, Mukherji SK. DiffusionWeighted MR Imaging ofGlobal Cerebral Anoxia. American Journal of Neuroradiology. 1999;20 (6):9991007.

11. K. Sonam, Nahid Akthar Khan, Pa N Gayathri et al., Clinical and magnetic resonance imaging findings in patients with Leigh syndrome and SURF1 mutations. Brain and Development 2014; 36 (9): 807-812.

12. Bonfante E, Koenig MK, Adejumo RB, Perinjelil V, Riascos RF. The neuroimaging of Leigh syndrome: case series and review of the literature. Pediatric radiology. 2016;46 (4):443-51.

13. Fujioka M, Okuchi K, Hiramatsu KI, Sakaki T, Sakaguchi S, Ishii Y. Specific changes in human brain after hypoglycemic injury. Stroke. 1997;28 (3):584-7.

14. Lim CC, Gan R, Chan CL, et al. Severe hypoglycemia associated with an illegal sexual enhancement product adulterated with glibenclamide: MR imaging findings. Radiology 2009;250 (3):193-201.

15. Bartynski WS, Boardman JF. Distinct imaging patterns and lesion distribution in posterior reversible encephalopathy syndrome. Am J Neuroradiol 2007;28 (6):1320-7.

16. Miller GM, Baker HL, Okazaki H, Whisnant JP. Central pontine myelinolysis and its imitators: MRfindings.Radiology 1988;168 (5):795-80

17. Babanrao SA, Prahladan A, Kalidos K, Ramachandran K. Osmotic myelinolysis: Does extrapontine myelinolysis precede central pontine myelinolysis? Report of two cases and review of literature. The Indian journal of radiology and imaging. 2015;25 (2):177.

18. Inoue $\mathrm{E}$, Hon $\mathrm{S}$, Narumi $\mathrm{Y}$, Fujita M, Kuriyama $\mathrm{K}$, Kadota $\mathrm{T}$ and Kuroda C. Portal-systemic encephalopathy: presence of basal ganglia lesions with high signal intensity on MR images. Radiology 1991; 179 (4):551-555

19. Pujol A, Graus F, Peri J, Mercader JM, Rimola A. Hyperintensity in the globus pallidus on TI-weighted and inversion-recovery MRI: a possible marker of advanced liver disease. Neurology 1991;41 (5): 1526- 
1527

20. Cheng A, Han L, Feng Y, Li H, Yao R, Wang D, Jin B. MRI and clinical features of maple syrup urine disease: preliminary results in 10 cases. Diagnostic and Interventional Radiology. 2017;23 (5):398.

21. Felber SR, Sperl W, Chemelli A, Murr C, Wendel U. Maple syrup urine disease: metabolic decompensation monitored by proton magnetic resonance imaging and spectroscopy. Ann Neurol 1993;33 (1): 396-401

22. Kim TJ, Kim IO, Kim WS, Cheon JE, Moon SG, Kwon JW, Seo JK, Yeon KM. MR imaging of the brain in Wilson disease of childhood: findings before and after treatment with clinical correlation. American journal of neuroradiology. 2006;27 (6):1373-8.

23. Kalita J, Misra UK. Comparison of CT scan and MRI findings in the diagnosis of Japanese encephalitis. Journal of the neurological sciences. 2000;174 (1):3-8.

24. Dung NM, Turtle L, Chong WK, Mai NT, Thao TT, Thuy TT, Kneen R, Phu NH, Wills B, Farrar J, Das K. An evaluation of the usefulness of neuroimaging for the diagnosis of Japanese encephalitis. Journal of neurology. 2009;256 (12):2052.

25. Misra UK, Kalita J, Phadke RV, Wadwekar V, Boruah DK, Srivastava A, Maurya PK, Bhattacharyya A. Usefulness of various MRI sequences in the diagnosis of viral encephalitis. Acta tropica. 2010;116 (3):206-11.

26. Bhoi SK, Naik S, Kumar S, Phadke RV, Kalita J, Misra UK. Cranial imaging findings in dengue virus infection. Journal of the neurological sciences. 2014;342 (1-2):3641.

27. Soni BK, Das DS, George RA, Aggarwal R, Sivasankar R. MRI features in dengue encephalitis: A case series in South Indian tertiary care hospital. The Indian journal of radiology and imaging. 2017;27 (2):125.

28. García-Santos JM, del Río ST, Sánchez A, MartínezLage JF. Basal ganglia and thalamic tumours: an imaging approximation. Child's Nervous System. 2002;18 (8):412-25.

29. Fu W, Ju Y, Zhang S, You C. Pediatric Basal Ganglia Region Tumors: Clinical and Radiologic Features Correlated with Histopathologic Findings. World neurosurgery. 2017;103 (6):504-16.

30. Raz E, Kapilamoorthy TR, Gupta AK, Fiorelli M. Case 186: Dysembrioplastic Neuroepithelial Tumor. Radiology. 2012;265 (1):317-20.

Source of Support: Nil; Conflict of Interest: None

Submitted: 01-07-2018; Accepted: 02-08-2018; Published online: 13-08-2018 